\title{
The Combined Prognostic Significance of Alkaline Phosphatase and Intracranial Arterial Calcifications in Hemodialysis Patients
}

\author{
Daniel Erez ${ }^{a}$ Feda Fanadkab Sydney Benchetrit ${ }^{c, d}$ Keren Cohen-Hagai ${ }^{c, d}$ \\ aDepartment of Internal Medicine D, Meir Medical Center, Kfar Saba, Israel; 'bepartment of Radiology, Meir Medical \\ Center, Kfar Saba, Israel; 'Department of Nephrology and Hypertension, Meir Medical Center, Kfar Saba, Israel; \\ ${ }^{\mathrm{d}}$ Sackler Faculty of Medicine, Tel Aviv University, Tel Aviv, Israel
}

\section{Keywords}

Vascular calcifications · Hemodialysis · Alkaline phosphatase · Mineral bone disease

\begin{abstract}
Introduction: The prevalence of intracranial arterial calcification (ICAC) in maintenance hemodialysis (MHD) patients is about $90 \%$, and its severity is correlated with age, hemodialysis vintage, and mineral bone disease. Elevated concentrations of calcium and phosphorus are not sufficient for medial calcification because of inhibition by pyrophosphate. Alkaline phosphatase (ALP) promotes calcification by hydrolyzing extracellular pyrophosphate. Epigenetic mechanisms involving ALP inhibition by apabetalone were investigated as a potential target for preventing vascular calcifications (VCs). This study assessed the combined impact of VCs and elevated serum ALP on mortality among chronic HD patients. Methods: VCs represented by ICAC were measured simultaneously with mineral bone disease parameters including serum ALP of MHD patients who underwent noncontrast brain computed tomography from 2015 to 2018 in our institution. Results: This retrospective study included 150 MHD patients (mean age $71.3 \pm 12.1$ years, $60.1 \%$ male).
\end{abstract}

Of the total cohort, $12(7.8 \%)$ had no brain calcifications and $69(45.1 \%)$ had multiple intracranial calcifications. Considering the patients with normal ALP and no calcification as the reference group yielded adjusted odds ratios for all-cause mortality of 4.6 ( $95 \% \mathrm{Cl}: 1.7-12.7)$ among patients with brain calcifications and normal ALP $(p=0.003)$ and odds ratios for all-cause mortality of 6.1 (95\% Cl: $2.1-17.7)$ among patients with brain calcifications and elevated ALP $(p=0.001)$. Conclusion: We found an independent association between ICAC and the risk of death among MHD patients. The combined effect of ICAC and elevated ALP was associated with a higher odds ratio for all-cause mortality in MHD patients and may contribute to the risk stratification of these patients.

(c) 2021 The Author(s).
Published by S. Karger AG, Basel

\section{Introduction}

Mineral and bone disorder (MBD) is a systemic disorder of mineral and bone metabolism characteristics of patients with chronic kidney disease (CKD). It is manifested by vascular or other tissue calcifications and is associated with increased risk of vascular and all-cause mortality, especially among patients undergoing maintenance he- karger@karger.com www.karger.com/ajn

Karger $\stackrel{\text { ' }}{5}$

GOPEN ACCESS
(C) 2021 The Author(s)

Published by S. Karger AG, Basel

This is an Open Access article licensed under the Creative Commons Attribution-NonCommercial-4.0 International License (CC BY-NC) (http://www.karger.com/Services/OpenAccessLicense), applicable to the online version of the article only. Usage and distribution for commercial purposes requires written permission.
Correspondence to:

Keren Cohen-Hagai, keren.cohen@ clalit.org.il 
modialysis (MHD) [1]. Previous reports have shown that vascular calcifications (VCs) are 5-10 times more common in MHD patients than in age-matched nondialysis control subjects, with a prevalence of $25-90 \%$ [2]. Among MHD patients, serum alkaline phosphatase (ALP) is increased in high-turnover bone disease [3]. Elevated ALP levels are associated with secondary hyperparathyroidism, renal osteodystrophy, and cardiovascular disease. In addition, serum parathyroid hormone (PTH) and phosphate are associated with all-cause mortality $[1,3]$. Several studies demonstrated that hyperphosphatemia is closely associated with advanced VC in MHD patients and that a high serum PTH level is a potent predictor of progression of coronary artery calcification $[3,4]$. The National Kidney Foundation Kidney Disease Outcome Quality Initiative Guidelines on CKD MBD recommend target ranges for serum calcium, phosphorus, and PTH concentrations in CKD patients from 2003 [5]. Yet, these guidelines do not provide a target range for ALP, although it is usually measured in MHD patients. Unlike serum PTH that originates from the parathyroid glands and affects bone metabolism, serum ALP originates from the bone itself and affects bone metabolism $[1,6,7]$. Previous studies demonstrated an incremental linear relationship between serum ALP levels and mortality among MHD patients $[3,8,9]$. This positive correlation is probably related to VCs through a pyrophosphate link, where ALP seems to play a mediating role in hydrolyzing and inactivating organic pyrophosphate $[10,11]$.

Elevated concentrations of calcium and phosphorus are not sufficient for medial calcification because of inhibition by pyrophosphate. ALP promotes calcification by hydrolyzing pyrophosphate, and epigenetic mechanisms involving its inhibition by apabetalone were investigated as a potential target for preventing $\mathrm{VC}$ [12].

Although a positive correlation between ALP levels and mortality has been documented, the interplay between ALP and VC defined by imaging modalities has been less frequently assessed. A few studies examined the correlation between VC using spiral computed tomography $(\mathrm{CT})$, ALP levels, and mortality $[13,14]$. In a retrospective study including 587 patients initiating hemodialysis in a single center [14], VCs were assessed at dialysis initiation using the aortic calcification index (ACI) and ALP levels, and the association between ALP and VC was analyzed. Patients with ACI-high ALP had the greatest risk for cardiovascular events and mortality. Another study described increased frequency of intra- and extracranial cerebrovascular calcifications in MHD patients [15]. However, the correlation between ALP levels and cerebrovascular calcifications and the interplay between them have not been examined. This study assessed the combined impact of VC and elevated serum ALP on mortality, among MHD patients.

\section{Methods}

This retrospective cohort study included MHD patients who had undergone brain noncontrast CT from January 1, 2015 to December 31, 2018 at Meir Medical Center. MHD was defined as a minimum of 3 months of hemodialysis. Most of the MHD patients were treated using conventional HD schedules (thrice weekly sessions, $4 \mathrm{~h}$ each), with high-flux membranes in the same dialysis unit. Hypertension, diabetes mellitus, and heart disease were determined based on a documented diagnosis in the patient's medical record.

The patients included in this cohort underwent brain CT for various clinical indications, as decided by their physician. Most were for evaluation in the emergency department after a trauma or fall, to enable the use of anticoagulation during hemodialysis.

\section{Serum ALP}

Serum ALP levels were measured in a central laboratory using an automatic analyzer method. The normal reference range of serum ALP is $30-120 \mathrm{U} / \mathrm{L}$. Since ALP is an enzyme found in additional tissues, except bone (mainly the liver and bile duct) and this study aimed to assess the effect of the bone ALP, we measured alkaline aminotransferase simultaneously and excluded all patients with an abnormal alkaline aminotransferase level that suggested the liver or bile duct origin of ALP). The specific ALP bone isoenzyme test was not available for clinical use in our institute during the study period.

\section{Calcification Scoring}

A neuroradiologist, who was blinded to the clinical and laboratory information of the study sample, performed repeated and directed radiologic assessments of brain CTs and used 2 methods to score the intracranial arterial calcifications [16]. Assessment of the calcifications included scoring calcifications of the common carotid arteries from 0 to 4 as follows: 0 , no calcifications; 1 , calcification $1 \mathrm{~mm}$ thick or stippled; 2, calcification $2 \mathrm{~mm}$ thick, thin continuous, or thick discontinuous; 3 , calcification $3 \mathrm{~mm}$ thick or thick continuous; and 4-calcification $>3 \mathrm{~mm}$ thick or double tracts.

We also assessed intracranial arterial calcifications by scoring the extent of the calcification in the circumference of the left and the right common carotid arteries, from 0 to 4 as follows: 0 , no calcification; 1 , dot of calcification; 2 , crescentic area of calcification spanning $<90^{\circ}$ of the carotid wall circumference; 3 , calcification spanning $90-270^{\circ}$ of the carotid wall circumference; 4 , calcification spanning $270-360^{\circ}$ of the carotid wall circumference. To simplify the figures and tables, we combined some of the groups for data analysis, such that patients with a score of 0 were intracranial arterial calcification (ICAC) $=0$, scores 1 and 2 were grouped as 1 , and scores of 3 and 4 were grouped as 2 .

\section{Ethical Considerations}

The study was approved by the Meir Medical Center Institutional Ethics Committee in keeping with the principles of the Declaration of Helsinki. 
Statistical Analysis

Data are presented as numbers and percentages for nominal parameters and as means and standard deviations for continuous parameters. Continuous variables were examined for normality (Shapiro-Wilk test), and data were analyzed accordingly. The $t$ test or one-way ANOVA was used for normally distributed variables, and the Mann-Whitney or Kruskal-Wallis test for nonparametric variables. A multivariate logistic regression model including all significant variables in the univariate analysis was applied to estimate odds ratios. Survival curves were obtained using the KaplanMeier method and compared using 2-sided log-rank statistics. $p<$ 0.05 was considered statistically significant. Data were analyzed using SPSS version 27 (IBM Corporation, Armonk, NY, USA).

\section{Results}

A total of 150 MHD patients were included in this study. Their mean age was $71.3 \pm 12.1$ years, and $60.1 \%$ were male. Further clinical information is detailed in Table 1 .

Of the total cohort, 12 (7.8\%) had no brain calcifications, 35 (22.9\%) had one intracranial calcification, and $103(67.3 \%)$ had $>2$ intracranial arterial calcifications, when calcifications were graded by the extent of the calcification in the circumference of the common carotid arteries (Table 1). When calcifications were assessed by the calcification thickness, only 11 patients had no calcifications in the common carotid artery and $>56 \%$ had a calcification $>2$ mm thick.

The median ALP level was $115 \mathrm{IU} / \mathrm{L}$, ranging from 31 to 780 (mean $151.1 \pm 122.8$ ). We did not find significant changes in ALP levels as a function of intracranial calcification scores $(p=0.491,0.725)$.

\section{All-Cause Mortality}

Mortality during the study period was $62.7 \%(n=94)$. Patients with normal ALP had better survival, as compared to patients with elevated ALP levels (mean survival of $413 \pm 62$ vs. $282 \pm 44$ days, respectively; $p=0.037$; Fig. 1a). We did not find a significant difference in survival as a function of ICAC score (Fig. 1b).

To assess the combined impact of VC and elevated ALP on mortality, we grouped the study cohort into 4 groups according to calcification and ALP (Table 2). Considering the patients with normal ALP and no calcification as the reference group, yielded adjusted odds ratio (OR) for all-cause mortality of 4.6 (95\% CI; 1.7-12.7) among patients with brain calcifications and normal ALP $(p=0.003)$, and OR for all-cause mortality of 6.1 (95\% CI; 2.1-17.7) among patients with brain calcifications and elevated ALP.

Alkaline Phosphatase and Calcifications in Hemodialysis
Table 1. Clinical and laboratory characteristics of the study sample $(n=150)$

\begin{tabular}{ll}
\hline Mean age \pm SD, years & $71.3 \pm 12.1$ \\
Male/female, $n$ & $92 / 58$ \\
Body mass index & $29.8 \pm 7.4$ \\
Dialysis vintage, months & $24.8 \pm 38$ \\
Comorbidities, $n$ (\%) & \\
$\quad$ Diabetes mellitus & $87(58)$ \\
$\quad$ Ischemic heart disease & $55(36.6)$ \\
$\quad$ Heart failure & $49(32.7)$ \\
Hypertension & $125(83.3)$ \\
Baseline laboratory values (desired range) & \\
$\quad$ Alanine transaminase, U/L & $18 \pm 15(0-40)$ \\
ALP, U/L & $151.1 \pm 122.8(30-120)$ \\
$\quad$ Total calcium, mg/dL & $8.4 \pm 0.8(8.5-10.5)$ \\
Phosphorus, mg/dL & $5.5 \pm 1.9(2.5-5.0)$ \\
Albumin, g/dL & $3.3 \pm 0.6(3.5-5.5)$ \\
PTH, pg/mL & $308.1 \pm 189.8(106-477)$ \\
Vitamin D 25OH, nmol/L & $45.3 \pm 21.1(75-125)$ \\
Vitamin D 1,25OH, nmol/L & $35.6 \pm 39.7(37-158)$ \\
Intracranial arterial calcification SCORE, $n(\%)$ \\
0 & $12(7.8)$ \\
1 & $35(22.9)$ \\
2 & $34(22.2)$ \\
3 & $36(23.5)$ \\
4 & $33(21.6)$ \\
Chronic medications, $n$ (\%) & $18(12)$ \\
Calcium-based phosphorus binders & $9(6)$ \\
Non-calcium-based phosphorus binders & $35(23)$ \\
Calcimimetics & \\
&
\end{tabular}

Intracranial arterial calcifications were graded by the extent of the calcification in the circumference of the left and the right common carotid arteries, as follows: 0 , no calcification; 1 , dot of calcification; 2 , crescentic area of calcification spanning $<90^{\circ}$ of the carotid wall circumference; 3 , calcification spanning $90-270^{\circ}$ of the carotid wall circumference; 4 , calcification spanning $270-360^{\circ}$ of the carotid wall circumference. ALP, alkaline phosphatase; PTH, parathyroid hormone.

Multivariate analysis showed that the most important predictors of all-cause mortality were ischemic heart disease and the combination of elevated ALP with ICAC ( Table 3). Treatment for MBD did not change these results.

\section{Discussion}

This retrospective study evaluated the association between ALP, VC and all-cause mortality among $150 \mathrm{MHD}$ patients. We found a significant increase in all-cause mortality among patients with ICAC and a further increase in patients with elevated ALP. 


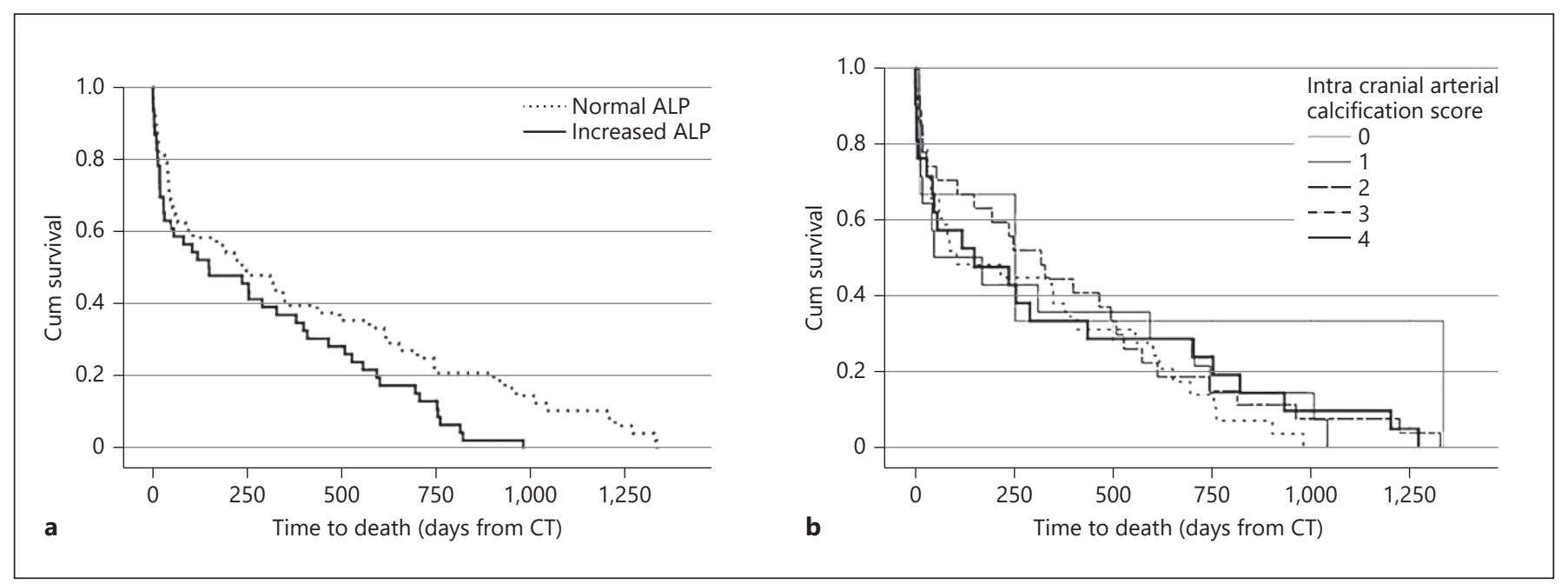

Fig. 1. a Survival plot of study sample as a function of serum ALP. Patients with normal ALP had better survival than patients with elevated ALP $(p=0.037)$. b Survival plot of study sample as a function of intracranial arterial calcification on CT. There was no significant difference in survival as a function of ICAC score using the KaplanMeier method $(p=0.570)$. CT, computed tomography; ICAC, intracranial arterial calcification; ALP, alkaline phosphatase.

Table 2. Odds ratio for all-cause mortality as a function of serum ALP and intracranial arterial calcifications on CT

\begin{tabular}{lllll}
\hline Variable & Odds ratio & \multicolumn{2}{l}{$95 \% \mathrm{Cl}$} & \multirow{2}{*}{$p$ value } \\
& & lower & upper & \\
\hline No ICAC, normal ALP & Reference group & & & 0.979 \\
No ICAC, elevated ALP & 1.0 & 0.3 & 3.3 & 0.003 \\
ICAC, normal ALP & 4.6 & 1.7 & 12.7 & 0.001 \\
ICAC, elevated ALP & 6.1 & 2.1 & 17.7 & \\
\hline
\end{tabular}

$\mathrm{Cl}$, confidence interval; ICAC, intracranial arterial calcification; ALP, alkaline phosphatase; CT, computed tomography.

Table 3. Multivariate analysis model for predictors of all-cause mortality

\begin{tabular}{lllll}
\hline Variable & Odds ratio & \multicolumn{2}{l}{$95 \% \mathrm{Cl}$} & \multirow{2}{*}{$p$ value } \\
\cline { 3 - 4 } & & lower & upper & \multirow{2}{*}{$\mathbf{0 3 3}$} \\
\cline { 1 - 2 } Ischemic heart disease & 3.5 & 1.1 & 11.2 & 0.07 \\
Heart failure & 2.5 & 0.9 & 6.9 & 0.9 \\
Hypertension & 1 & 0.2 & 4.4 & 0.07 \\
ALP & 1 & 0.9 & 1.1 & 0.9 \\
PTH & 1 & 0.2 & 4.6 & 0.2 \\
ICAC & 2.7 & 0.7 & 10.3 & $\mathbf{0 . 0 3}$ \\
ICAC and elevated ALP & 11.5 & 1.3 & 103 & \\
\hline
\end{tabular}

$\mathrm{Cl}$, confidence interval; ALP, alkaline phosphatase; ICAC, intracranial arterial calcification; PTH, parathyroid hormone. 
The association between VC and mortality among hemodialysis patients is well-established [17]. Cardiovascular disease is the major cause of death in patients with ESRD, and VC is a prognostic marker for death and cardiovascular events $[17,18]$. A previous meta-analysis showed that the presence of VC increased the risk of cardiovascular mortality by $181 \%$ and all-cause mortality by $73 \%$ in dialysis patients [19]. Risk factors for VC include age, dialysis vintage, diabetes mellitus, lipid levels, and inflammation. In addition, mineral and metabolic abnormalities play an important role in the development of VC. These include hyperphosphatemia, increased fibroblast growth factor-23, and elevated PTH levels [20, 21].

Altered patterns of mineral metabolism, including increased ALP, are often observed in ESRD. ALP is a hydrolase enzyme responsible for removing phosphate groups from many types of molecules, a process known as dephosphorylation. Serum ALP levels are usually elevated $(>120 \mathrm{U} / \mathrm{L})$ in patients with renal osteodystrophy, especially in those with high-turnover bone disease. Current data suggest that the magnitude of ALP elevation may be a more reliable marker of high-turnover bone disease than are PTH levels, because it originates directly from the pathologic bone system $[13,14,20]$.

Previous studies have indicated a link between ALP and VC $[13,22,23]$. Shantouf et al. [22] found a link between coronary artery calcifications and tissue ALP. In a retrospective analysis, Kim et al. [14] examined the combined prognostic significance of ALP and VC using the ACI. Similar to our study, the patients were stratified into 4 groups according to the degree of VC and ALP level. During a median follow-up of 3.1 years, patients with high ACI-high ALP had the greatest risk for the composite outcome of cardiovascular events and mortality (adjusted hazard ratio 2.25), while those with high ACI-low ALP also demonstrated increased risk for adverse outcomes (hazard ratio 2.09). Of note, intracranial calcifications were not assessed. Although the cohort in our study was smaller, relative risk for mortality was higher, both in the ICAC-normal ALP group (hazard ratio 4.6) and in the ICAC-high ALP group (hazard ratio 6.1). This study supports our results.

Since, as noted before, VC is related to increased risk for cardiovascular mortality, it would be reasonable to assume that elevated ALP in patients with ESRD would be associated with adverse outcomes. Indeed, the results of our study are in accordance with previous studies that demonstrated decreased survival in dialysis patients, related to elevated ALP levels. In their observational multicenter prospective study, Blayney et al. [8] showed that

Alkaline Phosphatase and Calcifications in Hemodialysis elevated ALP levels in both baseline and time-dependent models were associated with increased risk for all-cause cardiovascular and infection-related mortality, as well as with more fractures and parathyroidectomy. In another very large retrospective, 3-year cohort study including 74,000 hemodialysis patients [3], an increase in ALP by $10 \mathrm{U} / \mathrm{L}$ during the first 6 months was associated with increased risk for death during the subsequent 2.5 years.

We assessed ALP levels at baseline only. Patients were not followed up throughout the study. Therefore, due to the study design, we can only indicate associations and not causal relations between ALP, VC, and mortality among MHD. As noted in previous studies, ALP accelerates the deleterious effect of $\mathrm{VC}$ and, as such, has a fundamental role in its pathogenesis. Although clinical guidelines clearly recommend target ranges for calcium, phosphorus, and PTH levels, there is no such range for ALP levels, even though they are measured routinely. In fact, the magnitude of ALP may be a more reliable marker of renal osteodystrophy than PTH levels, as shown in a recent meta-analysis [24]. Moreover, in a retrospective analysis, Regidor et al. [3] demonstrated increased mortality with increasing ALP during follow-up. This association persisted within 3 strata of intact PTH levels $(<150,150-300$, and $>300 \mathrm{pg} / \mathrm{mL})$.

It is likely that the association between ALP and mortality among dialysis patients is related to VC through its pyrophosphate link $[25,26]$. Calcifications do not form in normal vessels, even with elevated concentrations of calcium and phosphorus. Previous studies revealed that normal aortas produce a soluble inhibitor of calcification - pyrophosphate. Plasma levels of extracellular pyrophosphate (ePPi) are reduced in ESRD. ALP promotes calcification by hydrolyzing pyrophosphate, and epigenetic mechanisms involving its inhibition by apabetalone were investigated as a potential target for preventing VC [25-28]. Lomashvili et al. [28] demonstrated that normal ePPi levels were sufficient to prevent VC. In their animal model, mice lacking ectonucleotide pyrophosphatase phosphodiesterase, which synthetizes ePPi, experienced accelerated aortic calcification. Of note, ALP activity in vascular smooth muscle is increased in uremia and could further compound the systemic ePPi deficiency [26]. These studies support our finding of poorer survival among patients with elevated ALP, as compared to normal ALP.

Apabetalone is an oral inhibitor of bromodomains [29]. It has a beneficial effect on biological pathways that drive pro-calcific processes [30]. In CKD patients, apabetalone had favorable effects on an estimated glomerular filtration rate [31] and reduced circulating ALP [31, 32]. 
The BETonMACE investigators [33] recently suggested that inhibition of BET proteins by apabetalone reduces the number of hospitalizations for heart failure among diabetic patients with recent acute coronary syndrome. Yet, the fact that the BETonMACE study did not show a statistically significant decrease in the incidence of major adverse cardiovascular events (MACE) may suggest that additional factors contribute to cardiovascular morbidity and mortality $[12,33,34]$. Since VCs and elevated circulating ALP are highly prevalent in HD patients, further studies are warranted to assess the effect of apabetalone on clinical outcomes in these patients.

\section{Study Limitations}

This study had some limitations. First, it was retrospective and used a single-center database that might have led to selection bias of patients who did not have a brain CT. Second, it had a cross-sectional retrospective design with both exposure (ALP, phosphorus, calcium, PTH) and outcome (intracranial calcifications) examined at the same point. Third, we did not use the gold standard method for diagnosing brain pathology - magnetic resonance imaging because it is less routinely used in our institution, as is the bone specific isoenzyme, ALP. We included only patients with normal alanine aminotransferase levels, so we believe the elevated ALP is mainly secondary to bone and not to other sources such as the liver and bile duct.

However, despite these limitations, this study has several important clinical implications. Both ALP and noncontrast CT are routine examinations that are often performed on MHD patients. ALP is performed as part of routine follow-up within the dialysis unit, and this study, as well as others, emphasizes the importance of controlling MBD parameters, including ALP, to reduce VC and their associated morbidity and mortality. The combined effect of ICAC and elevated ALP was associated with higher OR for all-cause mortality among MHD patients. This may contribute to the risk stratification of these patients, which is strongly needed in this specific high-risk population.
In conclusion, we found an independent association between ICAC and the risk of death among hemodialysis patients. The combined effect of ICAC and elevated ALP was associated with higher OR for all-cause mortality among MHD patients. Future prospective studies comparing the dynamics of $\mathrm{VC}$ progression in $\mathrm{MHD}$ as a function of all MBD parameters, including ALP, and adjusting for potential confounders, should be considered.

\section{Acknowledgments}

We thank Faye Schreiber, MS, for editing the manuscript.

\section{Statement of Ethics}

The study was approved by the local Institutional Ethics Committee in keeping with the principles of the Declaration of Helsinki (MMC 0251-17). In accordance with Ministry of Health regulations, the Institutional Ethics Committee did not require written informed consent because data were collected anonymously from the electronic medical records without active patient participation.

\section{Conflict of Interest Statement}

The authors have no conflicts of interest to declare.

\section{Funding Sources}

The authors have no funding sources to declare.

\section{Author Contributions}

Conception and design of research: D.E., F.F., S.B., and K.C.H.; data collection: F.F. and K.C.-H.; analyzed data, interpreted results, and prepared figures: K.C.-H.; drafted manuscript: D.E., S.B., and K.C.-H.; edited and revised manuscript: D.E., S.B., and K.C.-H.; all authors approved the final version of the manuscript.

\section{Data Availability Statement}

Data are available on request from the authors.

\section{References}

1 Kovesdy CP, Ahmadzadeh S, Anderson JE, Kalantar-Zadeh K. Secondary hyperparathyroidism is associated with higher mortality in men with moderate to severe chronic kidney disease. Kidney Int. 2008;73:1296302.

2 Ribeiro S, Ramos A, Brandao A, Rebelo JR, Guerra A, Resina C, et al. Cardiac valve calcification in hemodialysis patients: role of cal- cium-phosphate metabolism. Nephrol Dial Transplant. 1998;13:2037-40.

3 Regidor DL, Kovesdy CP, Mehrotra R, Rambod M, Jing J, McAllister CJ, et al. Serum alkaline phosphatase predicts mortality among maintenance hemodialysis patients. J Am Soc Nephrol. 2008;19:2193-203.

4 Malluche HH, Blomquist G, Monier-Faugere MC, Cantor TL, Davenport DL. High para- thyroid hormone level and osteoporosis predict progression of coronary artery calcification in patients on dialysis. J Am Soc Nephrol. 2015;26:2534-44.

5 National Kidney Foundation. K/DOQI clinical practice guidelines for bone metabolism and disease in chronic kidney disease. Am J Kidney Dis. 2003;42(Suppl 3): S1-201. 
6 Kovesdy CP, Kalantar-Zadeh K. Bone and mineral disorders in pre-dialysis CKD. Int Urol Nephrol. 2008;40:427-40.

7 Magnusson P, Sharp CA, Magnusson M, Risteli J, Davie MW, Larsson L. Effect of chronic renal failure on bone turnover and bone alkaline phosphatase isoforms. Kidney Int. 2001;60:257-65.

8 Blayney MJ, Pisoni RL, Bragg-Gresham JL, Bommer J, Piera L, Saito A, et al. High alkaline phosphatase levels in hemodialysis patients are associated with higher risk of hospitalization and death. Kidney Int. 2008;74:655-63.

9 Owaki A, Inaguma D, Tanaka A, Shinjo H, Inaba S, Kurata K. Evaluation of the relationship between the serum alkaline phosphatase level at dialysis initiation and all-cause mortality: a Multicenter, Prospective Study. Nephron Extra. 2017;7:78-88.

10 Narisawa S, Harmey D, Yadav MC, O'Neill WC, Hoylaerts MF, Millán JL. Novel inhibitors of alkaline phosphatase suppress vascular smooth muscle cell calcification. J Bone Miner Res. 2007;22:1700-10.

11 O'Neill WC. Pyrophosphate, alkaline phosphatase, and vascular calcification. Circ Res. 2006;99:e2.

12 Gilham D, Tsujikawa LM, Sarsons CD, Halliday C, Wasiak S, Stotz SC, et al. Apabetalone downregulates factors and pathways associated with vascular calcification. Atherosclerosis. 2019;280:75-84.

13 Guo J, Zeng M, Zhang Y, Huang H, Yang G, $\mathrm{Xu} F$, et al. Serum alkaline phosphatase level predicts cardiac valve calcification in maintenance hemodialysis patients. Blood Purif. 2020;49:550-9.

14 Kim DW, Hwang SY, Nam YJ, Kim D, Shin SJ, Yoon HE. The combined prognostic significance of alkaline phosphatase and vascular calcification in patients with end-stage kidney disease. Nutr Metab Cardiovasc Dis. 2020;30:1476-83.

15 Iwasa Y, Otsubo S, Nomoto K, Yashiro N, Yajima A, Kimata N, et al. Prevalence of intracranial artery calcification in hemodialysis patients - A case-control study. Int Urol Nephrol. 2012 Aug;44(4):1223-8. http:// dx.doi.org/10.1007/s11255-011-0026-7.
16 Babiarz LS, Yousem DM, Wasserman BA, Wu C, Bilker W, Beauchamp NJ. Cavernous carotid artery calcification and white matter ischemia. AJNR Am J Neuroradiol. 2003;24: 872-7.

17 Foley RN, Murray AM, Li S, Herzog CA, McBean AM, Eggers PW, et al. Chronic kidney disease and the risk for cardiovascular disease, renal replacement, and death in the United States Medicare population, 1998 to 1999. J Am Soc Nephrol. 2005; 16:489-95.

18 London GM, Guerin AP, Marchais SJ, Metivier F, Pannier B, Adda H. Arterial media calcification in end-stage renal disease:impact on all-cause and cardiovascular mortality. Nephrol Dial Transplant. 2003;18:173140.

19 Wang Z, Jiang A, Wei F, Chen H. Cardiac valve calcification and risk of cardiovascular or all-cause mortality in dialysis patients: a meta-analysis. BMC Cardiovasc Disord. 2018;18:12.

20 Linefsky JP, O’Brien KD, Katz R, de Boer IH, Barasch E, Jenny NS, et al. Association of serum phosphate levels with aortic valve sclerosis and annular calcification: the Cardiovascular Health Study. J Am Coll Cardiol. 2011; 58:291-7.

21 Iwata S, Hyodo E, Yanagi S, Hayashi Y, Nishiyama H, Kamimori K, et al. Parathyroid hormone and systolic blood pressure accelerate the progression of aortic valve stenosis in chronic hemodialysis patients. Int J Cardiol. 2013;163:256-9.

22 Shantouf R, Kovesdy CP, Kim Y, Ahmadi N, Luna A, Luna C, et al. Association of serum alkaline phosphatase with coronary artery calcification in maintenance hemodialysis patients. Clin J Am Soc Nephrol. 2009;4:110614.

23 Chen NX, Moe SM. Pathophysiology of vascular calcification. Curr Osteoporos Rep. 2015;13(6):372-80.

24 Palmer SC, McGregor DO, Macaskill P, Craig JC, Elder GJ, Strippoli GF. Meta-analysis: vitamin $\mathrm{D}$ compounds in chronic kidney disease. Ann Intern Med. 2007;147:840-53.

25 Schoppet M, Shanahan CM. Role for alkaline phosphatase as an inducer of vascular calcification in renal failure? Kidney Int. 2008;73: 989-91.
26 Lomashvili KA, Garg P, Narisawa S, Millan JL, O'Neill WC. Upregulation of alkaline phosphatase and pyrophosphate hydrolysis: potential mechanism of uremic vascular calcification. Kidney Int. 2008;73:1024-30.

27 Lomashvili KA, Cobbs S, Hennigar RA, Hardcastle KI, O’Neill WC. Phosphate-induced vascular calcification: role of phosphate and osteopontin. J Am Soc Nephrol. 2004;15:1392-401.

28 Lomashvili KA, Narisawa S, Millán JL, O’Neill WC. Vascular calcification is dependent on plasma levels of pyrophosphate. Kidney Int. 2014;85:1351-6.

29 Picaud S, Wells C, Felletar I, Brotherton D, Martin S, Savitsky P, et al. RVX-208, an inhibitor of BET transcriptional regulators with selectivity for the second bromodomain. Proc Natl Acad Sci U S A. 2013;110:19754-9.

30 Nicholls SJ, Ray KK, Johansson JO, Gordon A, Sweeney M, Halliday C, et al. Selective BET protein inhibition with apabetalone and cardiovascular events: a pooled analysis of trials in patients with coronary artery disease. Am J Cardiovasc Drugs. 2018;18(2):109-15.

31 Kulikowski E, Halliday C, Johansson J, Sweeney M, Lebioda K, Wong N, et al. Apabetalone mediated epigenetic modulation is associated with favorable kidney function and alkaline phosphatase profile in patients with chronic kidney disease. Kidney Blood Press Res. 2018; 43(2):449-57.

32 Haarhaus M, Ray KK, Nicholls SJ, Schwartz GG, Kulikowski E, Johansson JO, et al. Apabetalone lowers serum alkaline phosphatase and improves cardiovascular risk in patients with cardiovascular disease. Atherosclerosis. 2019;290:59-65.

33 Nicholls SJ, Schwartz GG, Buhr KA, Wong N, Sweeny M, Ray KK, et al. BETonMACE investigators. Apabetalone and hospitalization for heart failure in patients following an acute coronary syndrome: A Prespecified Analysis of the BETonMACE Study. Cardiovasc Diabetol. 2021 Jan 7;20(1):13.

34 Ray KK, Buhr KA, Ginsberg HN, Johansson JO, Kalantar-Zadeh K, Kulikowski E, et al. Effect of apabetalone added to standard therapy on major adverse cardiovascular events in patients with recent acute coronary syndrome and type 2 diabetes. A randomized clinical trial. JAMA. 2020;323:1565-73. 